\title{
Was macht ein Zeichen zu einem Zeichen? ${ }^{1}$
}

Jean Zumstein

Das vierte Evangelium erweist dem Leser einen grossen Dienst. In seinem als Kommentar verfassten Schluss formuliert der implizite Autor die von ihm verfolgte Absicht, mit welcher er die Geschichte des Lebens, des Todes und der Auferstehung Jesu verfasste: «Auch viele andere Zeichen hat nun zwar Jesus vor den Jüngern getan, die nicht in diesem Buch geschrieben sind. Diese aber sind geschrieben, damit ihr glaubt, dass Jesus der Christus ist, der Sohn Gottes, und damit ihr durch den Glauben Leben habt in seinem Namen» (Joh 20,30-31). Auf Anhieb wird dem «Zeichen» eine erhebliche Bedeutung verliehen. Die erzählten Zeichen ermöglichen den Zugang zum Glauben und von daher zum Leben. Sie bilden die Vermittlung, die der christologischen Offenbarung erlaubt, ein Lebensereignis für die Adressaten des Evangeliums zu werden.

Auf diese Weise wird der Leser auf die entscheidende Bedeutung der Zeichen aufmerksam gemacht, um seinen Glauben zu reflektieren und zu strukturieren. Sobald er aber über diesen Sachverhalt informiert ist und versucht, das vorgeschlagene Programm in die Tat umzusetzen, stösst er auf eine Schwierigkeit. Joh 20,30-31 verweist nicht einfach auf die von Jesus vollendeten Zeichen, sondern auf eine Auswahl davon. Es gibt die erzählten und die nicht erzählten Zeichen: "Auch viele andere Zeichen hat nun zwar Jesus vor den Jüngern getan, die nicht in diesem Buch geschrieben sind.» Somit wird der Leser absichtlich mit einer Auswahl konfrontiert. Aber um welche Auswahl geht es? Welches sind die in 20,30-31 erwähnten Zeichen?

Handelt es sich, falls der nahe Kontext privilegiert wird, um die in der Ostergeschichte berichteten Ereignisse, d.h. die Entdeckung des leeren Grabes und die Erscheinungen des Auferstandenen? Die Hypothese mag bestechend sein: die verschiedenen Momente der Ostergeschichte wären nicht als Ereignisse betrachtet, die ihre Bedeutung in sich selbst hätten, sondern als Ereignisse, die auf etwas anderes hinweisen würden. Als Zeichen würden sie offenbaren, dass

\footnotetext{
1 Dieser kleine und unprätentiöse Essay ist Ingolf Dalferth als Zeichen der Dankbarkeit für die freundschaftliche Zusammenarbeit gewidmet. Obwohl eigentlich nur ein Systematiker und Religionsphilosoph die hermeneutische Frage des Zeichens sachgemäss reflektieren kann, haben einige neutestamentlichen Autoren, vor allem Johannes, mit der Arbeit schon begonnen ...
} 
der Gekreuzigte-Erhöhte lebendig ist und von nun an bei Gott lebt. Dieses göttliche Lebendig-Sein des Auferstandenen würde für den Leser die Relevanz der christologischen Offenbarung bekräftigen.

Gemäss einer anderen in der Kritik vertretenen Hypothese denkt der implizite Leser vor allem an die im Evangelium berichteten Wundergeschichten, die bei Johannes als «Zeichen» bezeichnet werden. Aus der Warte der Philologie spricht alles dafür. Aber darf man sich wirklich vorzustellen, dass der am Ende seiner Erzählung angelangte Autor nur die Wundergeschichten als wichtige Ereignisse des Lebens Jesu ausgewählt und sich ohne nähere Begründung der grossen Offenbarungsreden, der Passions- und der Ostergeschichte entledigt hätte?

Diese 〈Lightversion` der Zeichen überzeugt kaum und führt zu einer dritten Hypothese: Das ganze Schicksal Jesu mit seinen verschiedenen Aspekten soll als Zeichenreservoir betrachtet werden. Das heisst: Die Offenbarung ist nie unmittelbar zugänglich. Sie wird nur durch Vermittlungen greifbar, die einer Decodierung bedürfen. Gott kann nur in einer angemessenen Sprache - in der Sprache der Zeichen - zum Ausdruck kommen.

Diese Hypothese hat eine gewisse Attraktivität.Auch wenn sie nur als Hypothese zu betrachten ist, verschärft sie die Probleme. Denn der Schluss des Evangeliums lässt nicht nur die nähere Bestimmung des Inhaltes der Zeichen offen, sondern er gibt dem Leser auch keinen hermeneutischen Schlüssel in die Hand, der ihre Decodierung ermöglichen würde, damit sie zum Auslöser des Glaubens und zum Geber des Lebens werden.

Wie ist also die johanneische Erzählung zu entziffern, damit sie zum Zeichen wird? Ein Umweg über die sieben im vierten Evangelium aufbewahrten Wundergeschichten soll zu einer Vertiefung unserer Überlegungen führen, indem aufgedeckt wird, wie der implizite Autor die Zeichenproblematik reflektiert hat.

\section{Kana: Das unsichtbare Zeichen (Joh 2,1-11)}

Die Interpretation des Wunders zu Kana scheint zunächst mit keiner Schwierigkeit belastet $\mathrm{zu}$ sein. Es handelt sich um eine christologische Geschichte: Indem Jesus Wasser in Wein verwandelt, offenbart er seine Identität. Durch diese Handlung macht er auf den Anbruch der messianischen Zeit aufmerksam. Die Vollendung des Heils, die für das Ende der Geschichte erwartet wurde, geschieht in seiner Person hier und jetzt. 
Der Leser ist jedoch mit zwei Problemen konfrontiert. Einerseits zeigt die berühmte Weinregel von V. 10 («Jeder Mensch setzt zuerst den guten Wein vor, und wenn sie betrunken geworden sind, dann den geringeren; du hast den guten Wein bis jetzt aufbewahrt»), dass der Speisemeister das, was sich ereignet hatte, nicht wahrgenommen hat. Ein erstaunliches Wunder hatte sich vor seinen Augen abgespielt - sechshundert Liter Wasser wurden in Wein verwandelt! - und er hat nichts bemerkt. Er kostet das Getränk, ohne dessen Ursprung identifizieren zu können (V. 9-10: «Als aber der Speisemeister das Wasser kostete, das zu Wein geworden war, und nicht wusste, woher es war - die Diener aber, die das Wasser geschöpft hatten, wussten es -, da ruft der Speisemeister den Bräutigam und sagt zu ihm: Jedermann setzt zuerst den guten Wein vor, und wenn sie betrunken sind, den schlechteren»). Das Zeichen ist nicht evident. Gott handelt mitten in der Welt inkognito. Diejenigen, die mit dem Wirken Jesu unmittelbar konfrontiert sind, sind nicht in der Lage zu begreifen, was geschieht.

Das zweite durch die Erzählung gestellte Problem bestätigt diese Beobachtung. Die in V. 2 erwähnten Jünger sind vollkommen passiv. Sie sind zwar Zeugen der Handlung ihres Meisters, aber nicht deren Interpreten. Der Kommentar von V. 11 («Diesen Anfang der Zeichen machte Jesus zu Kana in Galiläa und offenbarte seine Herrlichkeit; und seine Jünger glaubten an ihn») gibt zu verstehen, dass nur der Erzähler, der den Gesichtspunkt des johanneischen Christus vertritt, den hermeneutischen Schlüssel besitzt, der die Decodierung der christologischen Handlung ermöglicht. Der auf dieses seltsame Wunder geworfene retrospektive Blick formuliert dessen genaue Tragweite. Die Verwandlung des Wassers in Wein ist als Zeichen zu verstehen, d.h. als eine Handlung, die auf Jesus als Vermittler der göttlichen Wirklichkeit verweist, wobei diese göttliche Wirklichkeit hier mit Überfülle, Freude und Fest assoziiert wird. Dadurch werden die Jünger zum Glauben eingeladen. Wohlgemerkt: Es handelt sich um die Jünger, die in Kap. 1 die Würde Jesu schon anerkannt haben. Für sie ist Kana nicht die Stunde der Entdeckung des Glaubens, sondern dessen Vertiefung und Strukturierung.

Trotz seiner Extravaganz zeigt Kana, dass das Wirken Gottes durch den johanneischen Christus keine unmittelbare Einsichtigkeit besitzt, die sich sofort durchsetzen würde. Erst der retrospektive Blick, der auf das Geschehnis geworfen wird durch denjenigen, der den johanneischen Code kennt, gibt dem Geschehenen Sinn. 


\section{Die späte Entdeckung des Zeichens (Joh 4,46- 54)}

Der königliche Beamte, der seinen Sohn retten will, ist auch in einen merkwürdigen Prozess involviert. Dieser Vater, der seinem sterbenden Kind helfen will, setzt auf Jesus, dessen Ruf als Heiler sich weit verbreitet hat. Er sucht ihn auf in der Hoffnung, ihn zu seinem Sohn führen zu dürfen. Sein Vertrauen wird zunächst nicht belohnt, denn das erste Wort, das Jesus an ihn richtet, ist ein Vorwurf: Wie die anderen Menschen glaubt er nicht, es sei denn er sehe ein Wunder (V. 48).

Der Grund für das Aufsuchen Jesu durch den königlichen Beamten ist aber gar nicht durch seinen Glauben motiviert, sondern durch die Sorge um sein sterbendes Kind. Es ist der johanneische Christus, der dieser Geschichte eine theologische Prägung verleiht. Der königliche Beamte nimmt die Aufforderung Jesu ernst, ohne dass Jesus ein weiteres Wort ausgesprochen hätte. Er vertraut ihm und geht nach Hause zurück. Wie in der Kanageschichte ist die durch das Wort "Geh hin! Dein Sohn lebt» vorausgesetzte göttliche Handlung aber nicht unmittelbar beobachtbar. Zu diesem Zeitpunkt der Erzählung wurde nur eine unverifizierbare Verheissung ausgesprochen.

Auf dem Heimweg löst die Begegnung zwischen dem königlichen Beamten und seinen Dienern einen Relektüre-Prozess aus. Der erhörte Vater entdeckt die Tragweite des Geschehenen, indem er den Verlauf der Ereignisse rekonstruiert. Erst seine interpretatorische Leistung führt zu einerVerbindung zwischen der Heilung des Kindes und der Verheissung Jesu.Von daher kann er auf dem Hintergrund des am Anfang der Erzählung formulierten Vorwurfs Heilung und Glaube assoziieren, d.h. er kann die Heilung als Manifestation der rettenden Macht Gottes verstehen, so dass die Szene - wie in Kana - mit der Entdeckung des christologischen Glaubens endet.

In diesem zweiten Kana-Zeichen hat das Wirken Jesu - wie bei dem Weinwunder - keinen Evidenzcharakter. Es kann nicht beobachtet werden. Erst die interpretatorische Arbeit des Vaters setzt die angekündigte Heilung in Verbindung mit der Verheissung Jesu und verwandelt dann diese Heilung in ein Zeichen, welches zum Glauben ruft. Der Ermöglichungsgrund der verstehenden Anamnese des Vaters ist aber die prophetische Aufforderung des johanneischen Christus. 


\section{Der undankbare Paralytiker (Joh 5,2-16)}

Das dritte johanneische Zeichen ist die Heilung eines Gelähmten am Teich Bethesda (Joh 5,2-16). Im Vergleich mit den zwei ersten Zeichen stürzt diese Erzählung den Leser in die Welt der Auseinandersetzung und der Undankbarkeit.

Das Verhalten des Paralytikers verdient Aufmerksamkeit. Seine Präsenz am Teich bezeugt seinen Heilungswunsch. Die Schwierigkeit des Vorhabens und der brutale Wettbewerb, der sich täglich dort abspielt, haben aber bei dem Gelähmten jegliche Hoffnung zunichte gemacht. Somit zeigt er keine Erwartung und spricht, als Jesus ihm nahe kommt, keine Bitte aus. Dennoch wird er geheilt. So wie er diese Heilung nicht ersucht hat, so drückt er auch nicht die kleinste Dankbarkeit aus und stellt keine Überlegungen über das an, was geschehen ist.Vor den Juden, die zu ihm kommen, um ihn über seine plötzliche Heilung zu befragen, kann er nur seine volle Ignoranz bezüglich der Person des Heilers zugeben. Schlimmer noch, als er endlich die Identität seines Wohltäters erfahren hat, zögert er nicht, sie den Juden, die durch diese Handlung an einem Sabbat irritiert sind, zu kommunizieren.

Der geheilte Paralytiker weist eine totaleVerschlossenheit in Bezug auf das, was ihm passiert ist, auf. Nirgends in der Erzählung wird die Heilung - auch wenn sie unbestreitbar ist - zu einem Zeichen, das ihm erlauben würde, die Handlung Gottes durch seinen Gesandten zu entdecken. Der einzige Aspekt, der im Streitgespräch entfaltet wird, ist die wegen der Heilung erfahrene Unannehmlichkeit seitens der Juden. Und gerade in diesem Punkt ist der geheilte Gelähmte eine Figur der Undankbarkeit und des Unverständnisses: Er schiebt seinem Heiler die ganze Verantwortung zu.

Ebenso wenig wie der Paralytiker gelangen die Juden, Zeugen der Heilung, zur Erkenntnis der wahren Bedeutung dessen, was sich abgespielt hat. Ihre einzige Decodierung der Heilung ist nomistisch ausgerichtet. Die Deutung des Geschehenen wird gemäss den Vorschriften des Gesetzes etabliert und tendiert dazu, in der von Jesus ausgeführten Handlung eine Übertretung des Willens Gottes zu sehen. Die Blindheit ist vollkommen: Die nomistische Deutung des Wunders qualifiziert dieses barmherzige Eingreifen Gottes als eineVerletzung seiner Ehre. Der gewählte Code für die Entzifferung des Wirkens Jesu ist irreführend und führt zu einer Umkehrung seines ursprünglichen Sinnes.

Dieses dritte Zeichen bestätigt die schon anhand der zwei ersten Zeichen hervorgehobene Perspektive. Das göttliche Wirken, das 
in den johanneischen Wundergeschichten zum Ausdruck kommt, entbehrt der Evidenz. Wie die Episode am Teich Bethesda es zeigt, kann die zeichenhafte Dimension vom jeweiligen Empfänger völlig ignoriert werden. Mehr noch: Die ehrwürdigen traditionellen religiösen Codes können zu einer Umdeutung führen.

\section{Das Brotwunder und die königliche Täuschung (Joh 6,1-15)}

Das Brotwunder schildert eine andere Variante des möglichen Missverständnisses der Zeichen, die sich ebenso katastrophal auswirkt. Zwar lässt das Glaubensbekenntnis des V. 14 ("Als nun die Leute das Zeichen sahen, das Jesus tat, sprachen sie: Dieser ist wahrhaftig der Prophet, der in die Welt kommen soll») zuerst einen angemessenen Ausweg des Erkenntnisprozesses seitens der Zeugen des Wunders erhoffen. Die erste Hälfte des Verses gibt nämlich zu bedenken, dass die versammelte Volksmenge diesmal im Brotwunder ein Zeichen wahrgenommen hat, d.h. eine Gabe, deren Urheber nur Gott sein kann. Ausserdem zieht die Volksmenge die zwingende Folgerung, indem sie die christologische Komponente des Ereignisses erkennt. Das Brotwunder verweist auf seinen Urheber, den johanneischen Christus, der als der endzeitliche Prophet identifiziert wird. Die Volksmenge hat eine Interpretationsarbeit durchgeführt, die sich als vollkommen sachgemäss erweist.

Dieses korrekte Glaubensbekenntnis löst aber in einer zweiten Phase eine fehlerhafte Handlung aus: «Da nun Jesus erkannte, dass sie kommen und ihn ergreifen wollten, um ihn zum König zu machen, zog er sich wieder auf den Berg zurück, er allein» (V. 15). Die messianische Würde, die Jesus zugeteilt wird, wird zu einer politischen und militärischen Funktion degradiert, der Gottesgesandte mit einer bekannten Figur verwechselt, die traditionsgemäss einen klaren Auftrag hat, nämlich das Volk Israel von der römischen Besatzung zu befreien und ihm Würde und Rang unter den Völkern wiederzugeben. Der göttliche Offenbarer wird instrumentalisiert, das Glaubensbekenntnis verwandelt sich in blosse Ideologie.

Schliesslich scheitert die Auslegung des Zeichens, auch wenn das Glaubensbekenntnis zur grössten Hoffnung Anlass gab. Das Scheitern ergibt sich nicht aus der Feindschaft oder dem Unglauben, sondern es wird durch die Unfähigkeit ausgelöst, den durch die Tradition angekündigten Propheten richtig zu identifizieren. Die traditionellen religiösen Vorstellungen erweisen sich als ambivalent. Sie führen 
zur Wahrheit, um diese nachher sofort wieder zu verschleiern. Die Interpretationsarbeit scheint schwierig und anspruchsvoll zu sein.

\section{Die Epiphanie auf dem Wasser (Joh 6,16-21)}

Der Gang des johanneischen Christus auf dem See richtet sich nur an die Jünger. Er bildet für sie ein Zeichen, das die Autorität Gottes über die Schöpfung bezeugt. Indem der johanneische Christus die berühmte Formel «Ich bin es, fürchtet euch nicht!» (V. 20) ausspricht, zeigt er, dass er die in der alttestamentlich-jüdischen Tradition Gott vorbehaltene Rolle übernimmt. Als Gottesgesandter unter den Menschen besitzt er von nun an die Vorrechte, die Gott gehören. Diese Autorität Christi hat eine soteriologische Ausrichtung: Sobald die durch den Sturm bedrängten Jünger ihren Herrn gesehen haben, legen sie am Ufer an. Sie sind von nun an in Sicherheit.

Wie in den schon erwähnten Wundergeschichten besitzt der Gang Jesu auf dem See keine Evidenz. Die einzige Reaktion der Jünger ist die Furcht (V. 19). Die Deutung des Zeichens wird durch die Personen der Erzählung nicht explizit formuliert. Sie muss vom Leser konstruiert werden. Nur derjenige, der die biblische Tradition ausführlich kennt und in Jesus den Gottesgesandten bekennt, kann die Erzählung als Epiphanie wahrnehmen.

Der Gang auf dem See steht im Kontrast zum Brotwunder. Diese Szene deutet an, was die Volksmenge nicht entdecken konnte. Sie führt nämlich zu einer entscheidenden näheren Bestimmung des Glaubensbekenntnisses. Die Jünger bleiben aber auf der Schwelle der Offenbarung. Es wird nie von den Jüngern gesagt, dass sie fähig waren, die Epiphanie richtig zu deuten oder dass sie schon im Besitz der Wahrheit gewesen wären. Es wird nur angedeutet, dass sie auf unerklärliche Weise das Ufer erreichen konnten.

\section{Der Blindgeborene und der Konflikt der Interpretationen (Joh 9)}

Die Kritik hat seit langem in dieser Episode eine symbolische Erzählung identifiziert, die zeigt, wie der in der Finsternis lebende Mensch das Licht erblickt, indem er Jesus begegnet. Vor allem zwei Merkmale beanspruchen die Aufmerksamkeit des Lesers. Erstens ist das Kap. 9 die einzige Erzählung, in der der johanneische Christus für eine beträchtliche Zeitspanne abwesend ist (von V. 8 bis zuV.34). 
Diese Abwesenheit symbolisiert die nachösterliche Zeit, in welcher die christliche Gemeinde sich während der Abwesenheit ihres Herrn gezwungen sieht, über die Bedeutung der geschehenen Zeichen zu debattieren. Zweitens wird die angemessene Decodierung der von dem johanneischen Christus durchgeführten Zeichen - hier die Heilung des Blindgeborenen - im Rahmen eines Konfliktes zwischen verschiedenen Perspektiven verfolgt, die alternative Deutungen des Geschehnisses anbieten.

Diese Vielfalt von antagonistischen Perspektiven kommt auf paradigmatische Weise zum Ausdruck. Zuerst löst der geheilte Blinde für die Nachbarn und Bekannten eine Krise der üblichen Wahrnehmung der Wirklichkeit aus (V. 8-12). Sie sind nicht mehr in der Lage zu deuten, was sich abgespielt hat. Folglich sehen sie sich gezwungen, sich an die Inhaber des Wissens zu wenden, um eine Antwort auf ihre Verlegenheit zu finden. Die Inhaber des Wissens die Pharisäer - werden auch in grosse Verlegenheit gebracht, als sie mit dem erzählten Ereignis konfrontiert werden, denn ihr Wissen ist nicht in der Lage, den ihnen vorgelegten Fall eindeutig zu deuten. Ihr Wissen gerät in eine Krise (V. 13-17). Wenn sie aber versuchen, sich auf ihre eigenen Referenzen zu stützen - ihre Auslegung der Tora - sind sie genötigt, die Wirklichkeit zu entstellen, damit diese mit ihrer Überzeugung übereinstimmt. Wie die Pharisäer im Kap. 5 gehen sie soweit, dass sie das göttliche Wirken als Übertretung des Gesetzes brandmarken (V. 24-33). Die religiösen Behörden, die sowohl das Wissen als auch die Macht in ihrem Besitz haben, scheitern in der Deutung der göttlichen Wirklichkeit, die sie zu ehren und zu bewahren behaupten. Die Eltern des geheilten Blinden verkörpern schliesslich eine noch andere Variante: Ihre Deutung der Fakten wird durch ihr unmittelbares Interesse und durch ihre Furcht vor den Juden bestimmt (V. 18-23).

Der Einzige, der sich auf dem Weg einer sachgemässen Interpretation befindet, ist der geheilte Blinde. Die beiden Züge, die seinVerhalten kennzeichnen, sind das Geständnis seiner Unwissenheit (er weiss nichts und folglich ist er offen für jegliche sachgemässe Erklärung) und seine intellektuelle Redlichkeit. Ohne zu schwanken unterbreitet er seinen Gesprächspartnern einen genauen und detaillierten Bericht seiner Heilung. Diese intellektuelle Redlichkeit erlaubt es ihm, sich zwar auch auf die religiöse Tradition zu berufen, aber nicht um Jesus zu verurteilen, sondern um sich seiner wahren Identität ahnungsvoll anzunähern. Der sich abspielende Konflikt ermöglicht es ihm, sich durch sukzessive Stufen in die Richtung der Wahrheit zu bewegen, ohne jedoch völlig das Ziel zu erreichen. 
Der einzige qualifizierte Interpret der Zeichen bleibt der johanneische Christus selbst. Wie der hermeneutische Prolog (V. 3-5) und die Offenbarungserklärung am Ende (V. 37) es zeigen, befindet sich der hermeneutische Schlüssel, der zu einem vollen Verständnis der Zeichen führt, bei demjenigen, der sie durchführt.

Die Heilung des Blindgeborenen demonstriert noch einmal, dass die Handlungen des johanneischen Christus mit keiner unmittelbaren, unumstrittenen Bedeutung versehen sind. Im Gegenteil: Die Taten Jesu lösen einen ausdauernden Interpretationskonflikt aus. Kein menschlicher Akteur hat das letzte Wort in diesem Konflikt. Nur der johanneische Christus ist in der Lage, dem Leser die Fähigkeit zu geben, seine Zeichen als Offenbarung seiner Identität wahrzunehmen. Die Bedeutung der Zeichen kann nicht erobert werden, sie wird immer wieder gegeben und kann nur im Glauben empfangen werden.

\section{Die Auferstehung des Lazarus und das Kreuz (Joh 11)}

Die Geschichte der Auferstehung des Lazarus ist mit der bis jetzt skizzierten Perspektive völlig kohärent. In dieser grossen Erzählung, in der zum ersten Mal die Interpretation dem Zeichen vorangeht, ist der johanneische Christus die einzige Figur, die in der Lage ist, den Sinn des Lebens und des Todes auf endgültige Weise zu formulieren. Diesmal richtet er sich nicht an theologische Behörden, die um die Verteidigung ihrer religiösen Tradition besorgt sind, sondern an "seine Freunde», d.h. an die Glaubenden jeder Generation. Die Autorität Gottes über das Leben und den Tod wird zur Wirklichkeit, die hier und jetzt sowohl im Wort des johanneischen Christus (V. 25: «Ich bin die Auferstehung und das Leben; wer an mich glaubt, wird leben, auch wenn er gestorben ist») als auch in seinem Wirken (V. 43: «Lazarus, komm heraus!») geschieht. In diesem letzten grossen Zeichen ist der johanneische Christus von Anfang bis Ende der Hermeneut seiner Handlung.

Das neue Element ist mit der Gestaltung des Plots gegeben. Das grösste Wunder, das die grösste Vollmacht offenbart, löst nicht eine dankbare Anerkennung, sondern die schroffe Verurteilung aus. Die religiösen Behörden lassen sich nicht überzeugen, sondern schicken denjenigen in den Tod, der der Geber des Lebens ist. Die Enthüllung der göttlichen Wirklichkeit durch den Gottesgesandten oder - anders formuliert - das letzte über die menschliche Existenz ausgesprochene 
Wort löst keine Begeisterung, keine Gefolgschaft, keine Anerkennung aus, sondern die gewaltige Verwerfung, die zum Kreuz führt.

Der durch die durchgeführten Zeichen entstehende Interpretationskonflikt, der Streit um ihre sachegemässe Auslegung bewirkt Gewalt und Hass.

\section{Schluss}

- Wie ist die zeichenhafte Geschichte Jesu in der nachösterlichen Zeit zu lesen? Wie ist die Gedächtnisarbeit durchzuführen? Die johanneische Erzählung gibt zu verstehen, dass die Wunder, für sich betrachtet, keine klare Bedeutung haben, sondern ein Rätsel darstellen. Nur derjenige, der den Code besitzt, ist in der Lage, in den Taten Jesu Zeichen wahrzunehmen. Das Zeichen ist weder unmittelbar zugänglich noch eindeutig. Der Begriff «Zeichen» setzt einen hermeneutischen Zusammenhang, der seine Decodierung ermöglicht.

- Die Lektüre des vierten Evangeliums führt zur Einsicht, dass die Interpretationscodes zahlreich sind. Sei es die Volksmenge, die religiösen Behörden oder der zum Glauben offene Mensch: Ihre Lektüren kreuzen sich und gehen auseinander. In der Abwesenheit Christi (vgl. Kap. 9) bleibt die Auslegung der Geschichte Jesu umstritten - man könnte sogar sagen: definitiv kontrovers.

- Der Interpretationskonflikt ist jedoch keineswegs unergiebig. In dieser Auseinandersetzung über die Zeichen Jesu kann jede Person der Erzählung ihre eigene Existenz noch einmal durchdenken und sich der entscheidenden Werte, die ihr Leben bestimmen, bewusst werden. Somit ist die Interpretation der Geschichte Jesu nicht nur eine kognitive Leistung, sondern auch ein existentielles Unterfangen, wo das Leben in seiner Ganzheit auf dem Spiel steht.

- Das Wunder - wenn es als Eingriff des Göttlichen in die menschliche Welt erfasst wird - ist nicht unmittelbar identifizierbar. Es taucht auf als eine Dekonstruktion der Gewissheiten, als eine Störung, die sich in die üblichen Weltanschauungen nicht leicht integrieren lässt. Es bewirkt Verlegenheit, Ignoranzgeständnisse und schliesslich Feindschaft. Das Wirken Jesu löst eine Krise der Erkenntnis aus.

- Nach Johannes ist allein Christus der wahre Hermeneut seiner Zeichen. Nur er besitzt den wahren Code. Durch seine Worte verleiht er der Erzählung ihre symbolische Tragweite und dadurch ermöglicht er dem Leser, eine neue Welt zu entdecken.

- Dr. Jean Zumstein ist Professor für Neutestamentliche Theologie, Exegese und Hermeneutik an der Universität Zürich. 